\title{
Lattice-Boltzmann simulations of three-dimensional fluid flow on a desktop computer Jeffrey D. Brewster*
}

\author{
United States Department of Agriculture, Agricultural Research Service, \\ Eastern Regional Research Center, 600 E. Mermaid Lane, Wyndmoor, PA 19038
}

Microbial Biophysics \& Residue Chemistry \& Core Technologies

U.S. Department of Agriculture, Agricultural Research Service

Eastern Regional Research Service, 600 East Mermaid Lane

Wyndmoor, Pennsylvania 19038

Abbreviations: LB, lattice-Boltzmann

*Corresponding author. Tel.: 215-233-6447; Fax: 215-233-6559; email:

jeffrey.brewster@ars.usda.gov 


\section{Mathematica Notebooks}

Two Mathematica notebooks, LBinit.nb and LBdemo.nb, are included. LBinit.nb contains definitions of simulation constants and variables, along with a large number of predefined functions for setting up, simulating, and displaying results. LBinit.nb must be evaluated before running the demonstration simulations. This is most conveniently done by saving the file as a Mathematica package, and importing it with the "<<" command. LBdemo.nb contains information on using the $\mathrm{C}$ simulation engine and contains sample simulations of various systems, including those in this report (small domains are used in the demo to avoid long delays). Procedures for setting up simulations, executing them, saving and retrieving data, and displaying the results data are illustrated. If you do not have access to Mathematica, the notebooks can be opened (but not executed) with the free viewer downloadable from http://www.wolfram.com/products/mathreader/.

\section{$\underline{\text { C Source Code }}$}

A C language program file, lb_d3q19.c, and a macro file, lb_d3q19.tm, are included. These files must be compiled and linked using the utilities, libraries and interface files included with the user's Mathematica software (these are unique to each version, operating system and processor). Complete instructions are provided in the Mathematica Mathlink documentation. An outline of the process is as follows:

- Create a folder/directory to contain the compiled program

- Copy lb_d3q19.c and lb_d3q19.tm to the program directory

- Copy the utility mprep, the interface file mathlink.h, and the library libML.a from the /Mathematica/AddOns/MathLink/DeveloperKit/YourOS/CompilerAdditions directory to the program directory

- $\quad$ Run the mprep utility with lb_d3q19.tm to create lb_d3q19.tm.c 
- Compile and link lb_d3q19.c, lb_d3q19.tm.c, mathlink.h and libML.a using your computer's $\mathrm{C}$ compiler to produce $\mathrm{lb}$ _d3q19 as an executable $\mathrm{C}$ program.

- Set the programDirectory variable in LBinit.nb to the directory where the executable lb_simis located

- Use the "Save As" command to save LBinit.nb in the Package Format as LBinit.m. It must be placed in one of the directories normally searched by Mathematica for packages, such as the user's home directory. 